\title{
Metallic Phase with Long-Range Orientational Order and No Translational Symmetry
}

\author{
D. Shechtman and I. Blech \\ Department of Materials Engineering, Israel Institute of Technology-Technion, 3200 Haifa, Israel \\ and \\ D. Gratias \\ Centre d'Etudes de Chimie Métallurgique, Centre National de la Recherche Scientifique, F-94400 Vitry, France \\ and \\ J. W. Cahn \\ Center for Materials Science, National Bureau of Standards, Gaithersburg, Maryland 20760
}

(Received 9 October 1984)

\begin{abstract}
We have observed a metallic solid ( $\mathrm{Al}-14-\mathrm{at} . \%-\mathrm{Mn}$ ) with long-range orientational order, but with icosahedral point group symmetry, which is inconsistent with lattice translations. Its diffraction spots are as sharp as those of crystals but cannot be indexed to any Bravais lattice. The solid is metastable and forms from the melt by a first-order transition.
\end{abstract}

PACS numbers: $61.50 . \mathrm{Em}, 61.55 . \mathrm{Hg}, 64.70 . \mathrm{Ew}$

We report herein the existence of a metallic solid which diffracts electrons like a single crystal but has point group symmetery $m \overline{3} \overline{5}$ (icosahedral) which is inconsistent with lattice translations. If the specimen is rotated through the angles of this point group (Fig. 1), selected-area electron diffraction patterns clearly display the six fivefold, ten threefold, and fifteen twofold axes characteristic ${ }^{1}$ of icosahedral symmetry (Fig. 2). Grains up to $2 \mu \mathrm{m}$ in size with this structure form in rapidly cooled alloys of Al with $10-14$ at.\% $\mathrm{Mn}, \mathrm{Fe}$, or $\mathrm{Cr}$. We will refer to the phase as the icosahedral phase. Microdiffraction from many different volume elements of a grain and dark-field imaging from various diffraction spots confirm that entire grains have longrange orientational order. If the orientational order decays with distance, its correlation length is far greater than the grain size. We have thus a solid metallic phase with no translational order and with with long-range orientational order.

The remarkable sharpness of the diffraction spots (Fig. 2) indicates a high coherency in the spatial interference, comparable to the one usually encountered in crystals. The diffraction data are qualitatively well fitted by a model consisting of a random packing of nonoverlapping parallel icosahedra attached by edges. ${ }^{2}$ The invariance of the local orientational symmetry from site to site and the finite number of possible translations between two adjacent icosahedra seem to be sufficient for insuring highly coherent interferences. Icosahedra are a common packing unit in intermetallic crystals with the smaller transition element at the center sur- rounded by twelve larger atoms arranged like the corners of an icosahedron. ${ }^{3}$ The symmetries of the crystals dictate that the several icosahedra in a unit cell have different orientations and allow them to be distorted, leaving the overall crystal consistent with the well-known crystallographic point and space groups. Even though icosahedral symmetry is of great importance as an approximate site symmetry in crystals, it cannot survive the imposition of lattice translations: Crystals cannot and do not exhibit the icosahedral point group symmetry.

Elementary crystallography indicates that fivefold

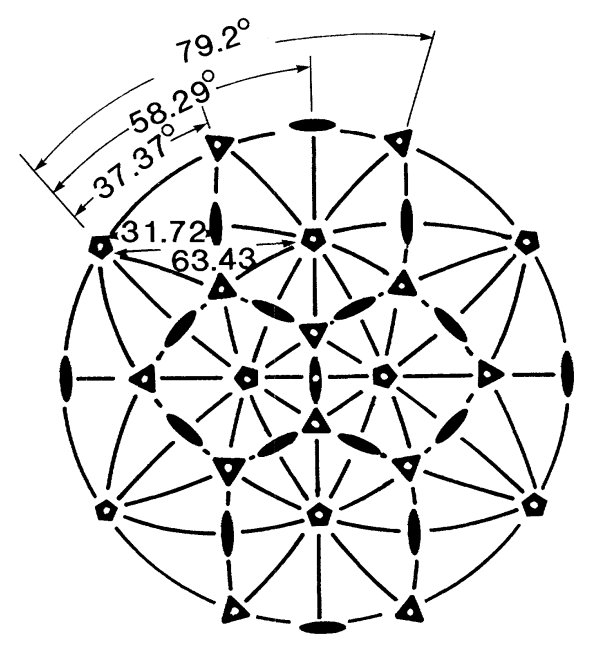

FIG. 1. Stereographic projection of the symmetry elements of the icosahedral group $m \overline{3} \overline{5}$. 


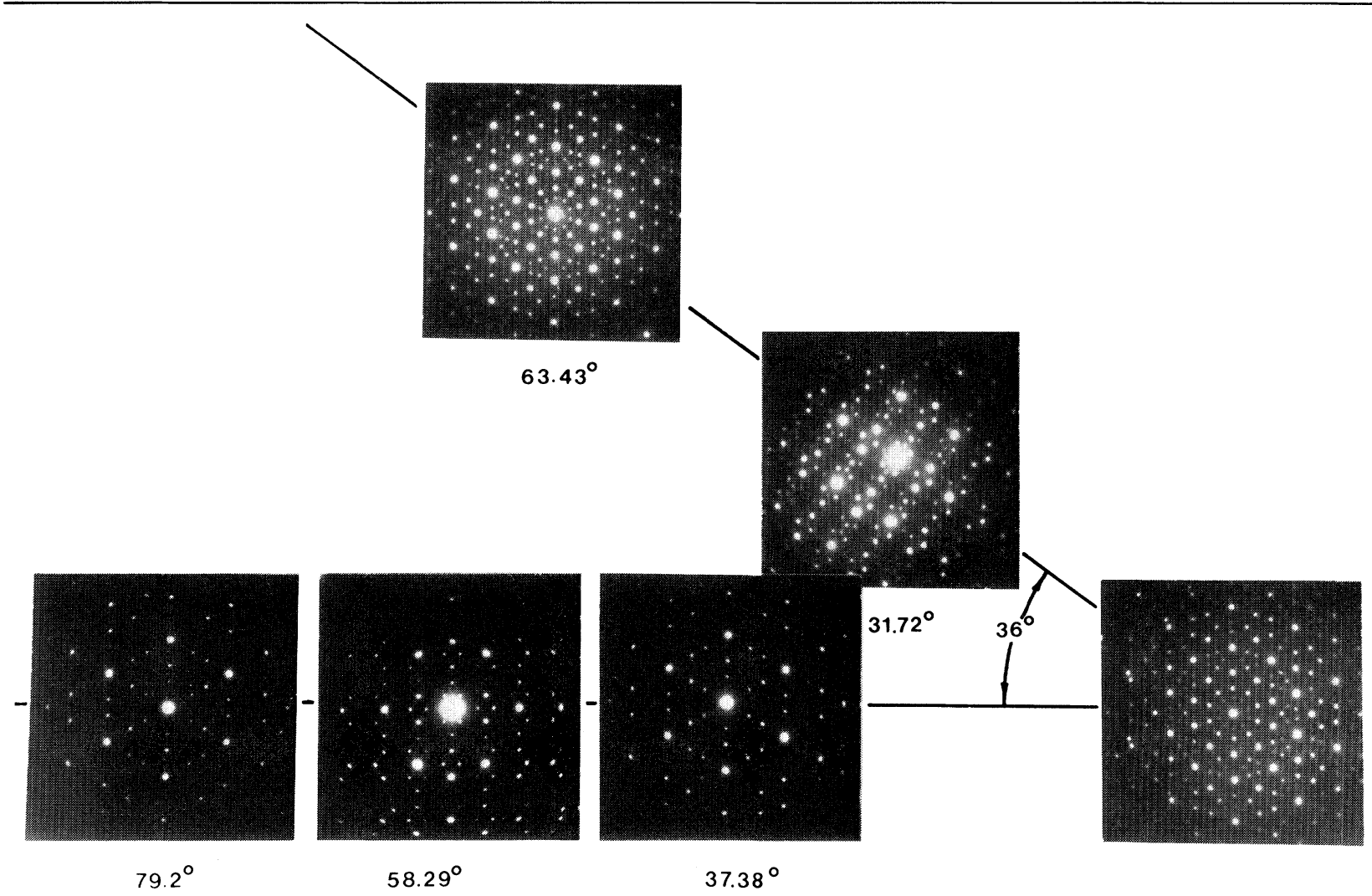

FIG. 2. Selected-area electron diffraction patterns taken from a single grain of the icosahedral phase. Rotations match those in Fig. 1.

axes are inconsistent with translational order. ${ }^{4}$ Crystals with an apparent fivefold axis do occur as multiple twins. ${ }^{5}$ Icosahedral symmetry could conceivably occur by multiple twinning, but at least five twins are required in order to obtain an icosahedral diffraction set from a conventional crystal. Experiments in the electron microscope and with $x$-ray diffraction contradict the twin hypothesis:

(1) A set of dark-field images taken from any reflection reveals no twins and in all cases the whole grain is illuminated.

(2) A convergent-beam diffraction pattern along the fivefold zone taken from an area $20 \mathrm{~nm}$ in diameter at any point of the grain displays all the reflections that appear in the selected-area diffraction pattern. This is also true when the thickness of the specimen is of the order of $10 \mathrm{~nm}$.

(3) An x-ray diffraction pattern ( $\mathrm{Cu} K \alpha$ source) was taken from a single-phase sample of the material containing many grains of various orientations. Had the phase consisted of a multiply twinned crystalline structure, it should have been possible to index the powder pattern regardless of the twins. The pattern obtained from the icosahedral phase could not be indexed to any Bravais lattice.

On the basis of these experiments we conclude that the icosahedral phase does not consist of multiply twinned regular crystal structures.

The icosahedral phase forms during rapid cooling of the melt by a nucleation and growth mechanism. This mechanism is characteristic of a first-order transition because the two phases coexist along a moving interface. Each particle nucleates at a center and grows out from there. The atomic rearrangements that result in the orientational order of the icosahedral phase occur at this interface, and the two adjoining phases differ in entropy and, for some alloys, in composition. If the transition were higher order, ordering would occur everywhere in the liquid instead of being confined to interfaces. Our evidence for the first-order character is morphological. We examined alloys with 10-14 at.\% Mn. Samples with $10 \%$ to $12 \%$ Mn showed many nodular grains separated from each other by crystalline films of fcc Al. The grains were approximately spherical in shape but were deeply indented with radial streaks composed of fcc Al crystals. The morphology is similar to a commonly observed one in rapid solidification in which crystals nucleate at 
many centers and grow until the remaining liquid solidifies, except that instead of isolated crystals we have isolated icosahedral grains embedded in solidified Al.

Another aspect of the first-order character of the transition is that segregation accompanies the growth of the icosahedral phase. In the $10 \%$ - and $12 \%-\mathrm{Mn}$ samples the growing phase rejects $\mathrm{Al}$ into the liquid. The indentations in the nodules are characteristic of the cellular morphology caused by an instability in the diffusion layer surrounding the solid resulting from segregation during growth. ${ }^{6}$ In the $14 \%$-Mn alloys the icosahedral grains occupy almost all of the volume of the specimen. Only small amounts of fcc $\mathrm{Al}$ occur along some grain boundaries where the grains grew to impingement. Some radial streaking still points to the nucleation centers from which the grains grew. We conclude that little segregation occurs in this alloy and that the icosahedral phase contains $14 \% \mathrm{Mn}$.

The obvious cellular morphology indicates that the growth of the icosahedral phase is slow enough to permit diffusional segregation on the scale of 1 $\mu \mathrm{m}$. With the diffusion coefficient in the liqud of order $10^{-8} \mathrm{~m}^{2} / \mathrm{s}$ this indicates interface velocities of order $10^{-2} \mathrm{~m} / \mathrm{s}$ and formation times of $10^{-4} \mathrm{~s}$. This is 2 to 3 orders of magnitude slower than the fastest known crystallization velocities of a metallic liquid during rapid cooling, but it is a typical upper limit of velocity of crystallization with composition changes. ${ }^{7}$ There is thus plenty of time for atomic rearrangements to occur at the interface between the melt and the icosahedral phase.

The icosahedral phase in rapidly solidified Al-Mn alloys is remarkably resistant to crystallization. Prolonged heating of $6 \mathrm{~h}$ at $300^{\circ} \mathrm{C}$ and $1 \mathrm{~h}$ at $350^{\circ} \mathrm{C}$ produced no detectable crystallization, but $1 \mathrm{~h}$ at $400^{\circ} \mathrm{C}$ caused crystallization to the stable $\mathrm{Al}_{6} \mathrm{Mn}$ phase. We conclude that the icosahedral phase is a truly metastable phase which nucleates and grows for a range of cooling rates which are slow enough to permit its formation but rapid enough to prevent crystallization, either from the melt or from the icosahedral phase after its formation.

The icosahedral phase has symmetries intermediate between those of a crystal and a liquid. It differs from other intermediate phases in that it is both solid, like a metallic glass, and that it has long-range orientational order. Many intermediate phases do have orientational order, but usually it is only local, and the transition to such phases is continuous. ${ }^{8,9}$ The possibility of an icosahedral phase with long range order was inferred from a computer simulation, ${ }^{10,11}$ and a first-order liquid-to-icosahedral phase transition has been predicted from a mean-field theory. ${ }^{11,12}$

We thank F. S. Biancaniello for spinning the alloys, C. R. Hubbard for x-ray experiments, and B. Burton for a critical review of the manuscript. This work was sponsored by the Defense Advanced Research Projects Agency and the National Science Foundation. This work was performed while one of us (D.S.) was a guest worker at the National Bureau of Standards and while another of us (D.G.) was at the Institute for Theoretical Physics, University of California at Santa Barbara.

${ }^{1}$ International Tables for Crystallography (Reidel, Higham, Mass., 1983), Vol. A, p. 179.

${ }^{2}$ D. Shechtman and I. Blech, to be published.

${ }^{3}$ F. C. Frank and J. S. Kasper, Acta Crystallogr. 11, 184 (1958), and 12, 483 (1959).

${ }^{4}$ M. J. Buerger, Elementary Crystallography (MIT Press, Cambridge, 1978).

${ }^{5} \mathrm{G}$. Friedel, Lecon de Crystallographie (Masson, Paris, 1921).

6W. W. Mullins and R. F. Sekerka, J. Appl. Phys. 35, 444 (1963).

${ }^{7}$ W. J. Boettinger, F. S. Biancaniello, G. Kalonji, and J. W. Cahn, in Rapid Solidification Processing II, edited by R. Mehrabian et al. (Claitors, Baton Rouge, 1980), p. 50. ${ }^{8}$ D. R. Nelson, Phys. Rev. B 28, 5515 (1983).

9J. Sadoc, J. Phys. (Paris), Colloq. 41, C8-326 (1980).

10P. J. Steinhardt, D. R. Nelson and M. Rouchetti, Phys. Rev. Lett. 47, 1297 (1981).

11P. J. Steinhardt, D. R. Nelson and M. Rouchetti, Phys. Rev. B 28, 784 (1983).

${ }^{12}$ A. D. J. Haymet, Phys. Rev. B 27, 1725 (1983). 


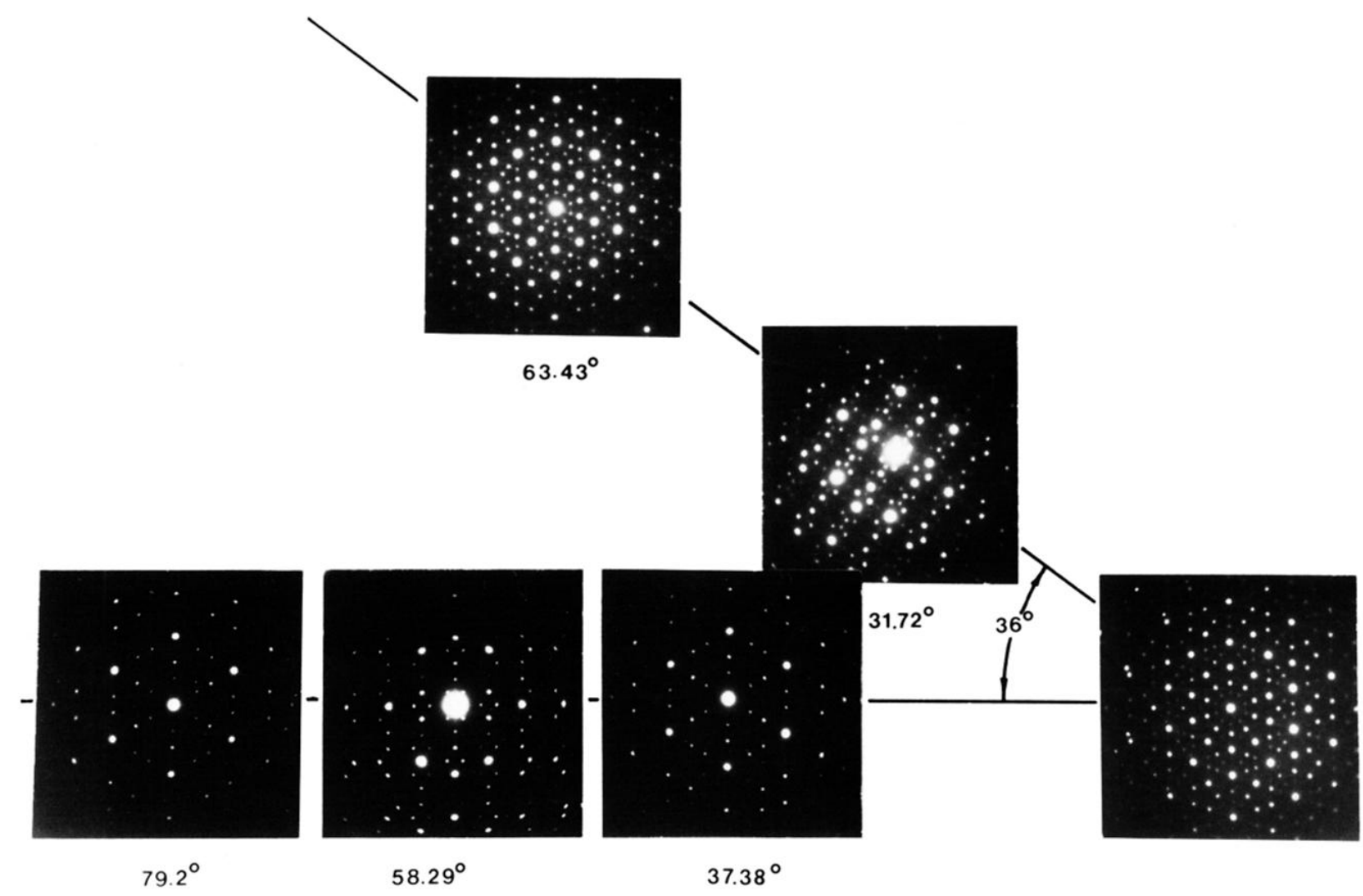

FIG. 2. Selected-area electron diffraction patterns taken from a single grain of the icosahedral phase. Rotations match those in Fig. 1. 